ESJ Social Sciences

\title{
Aprendizaje Sincrónico y Asincrónico del Año 2020: el Caso del Instituto Tecnológico de Saltillo, Coahuila
}

\author{
Leticia Urbina Valdes \\ Instituto Tecnológico Nacional de México, Campus Saltillo, México \\ Jesús Francisco Mellado Siller \\ Edith Reyes Ruiz \\ Universidad Autónoma de Coahuila, México
}

Doi:10.19044/esj.2022.v18n6p22

Submitted: 15 December 2021

Accepted: 08 February 2022

Published: 28 February 2022
Copyright 2022 Author(s)

Under Creative Commons BY-NC-ND

4.0 OPEN ACCESS

Cite As:

Urbina Valdes L., Mellado Siller J.F. \& Reyes Ruiz E. (2022). Aprendizaje Sincrónico y Asincrónico del Año 2020: el Caso del Instituto Tecnológico de Saltillo, Coahuila. European Scientific Journal, ESJ, 18 (6), 22. https://doi.org/10.19044/esj.2022.v18n6p22

\section{Resumen}

Esta investigación tiene como objetivo, encontrar las principales diferencias entre la educación sincrónica y la asincrónica, el estudio se llevó a cabo con las carreras de ingeniería del Instituto Tecnológico Nacional de México, Campus Saltillo, ubicado en el estado de Coahuila; se realizaron trabajos de investigación acerca de lo que son los antecedentes que se tienen registrados en las bases de datos, de ellas se obtuvieron las variables que se consideraron se deberían de medir en cada una de las formas de enseñanza; se realizó un análisis factorial para establecer el sustento de cuáles son las diferencias y si estas son significativas. Los resultados arrojaron en las saturaciones significativas de los factores y quedó de manifiesto, que si bien hay concordancias también existen diferencias en las maneras de enseñanza y éstas son los resultados importantes del estudio; ya que pueden ser consideradas en las planeaciones de estructuras de enseñanza tanto de forma presencial como de forma a distancia.

Palabras clave: Educación Asincrónica, Educación Sincrónica, Formas de aprendizaje 


\title{
Synchronous and Asynchronous Learning IN the Year 2020: The Case of the Technological Institute of Saltillo, Coahuila
}

\author{
Leticia Urbina Valdes \\ Instituto Tecnológico Nacional de México, Campus Saltillo, México \\ Jesús Francisco Mellado Siller \\ Edith Reyes Ruiz \\ Universidad Autónoma de Coahuila, México
}

\begin{abstract}
This paper focuses on identifying the main differences between synchronous and asynchronous education. The study was carried out at the National Technological Institute of Mexico, Campus Saltillo, which is located in the state of Coahuila. The research work was conducted based on the records that were registered in the databases, and the measured variables that were considered in the forms of education were obtained. A factorial analysis was utilized to establish what the differences are and if they are significant. The results showed the significant saturations of the factors and it became clear that although there are concordances, there are also differences in the ways of teaching. The results of the study should be considered in the planning of teaching structures both in person and remotely.
\end{abstract}

Keywords: Asynchronous Education, Synchronous Education, learning processes

\section{Introduction}

El presente trabajo se fundamenta en la tesis de maestría de la Maestra Leticia Urbina Valdés, y se trata de demostrar las principales diferencias entre dos grupos con diferentes tipos de acercamiento al conocimiento: el obtenido a partir de la utilización de herramientas de tecnología de Información y comunicación (TIC's) conocido como aprendizaje a Distancia y el acercamiento a la instrucción de tipo escolarizado conocido como presencial; las variables analizadas corresponden a las actividades que se tienen que hacer con el sistema escolarizado, y el sistema de enseñanza a Distancia. Este trabajo se realizó antes de la Contingencia del COVID-19 y se considera útil debido a que permite tener un acercamiento a las dos diferentes maneras de aprendizaje. El trabajo se realizó en el Instituto Tecnológico Nacional de México, Campus Saltillo, donde se obtuvo la muestra de los alumnos que participaban de las dos maneras de aprendizaje; Presencial y a Distancia, los resultados son interesantes debido a que con un Análisis Factorial aplicado a un instrumento validado se obtuvieron resultados que arrojan que existen 
diferencias en cuanto a las variables analizadas en cada uno de los grupos. Es una investigación que se inserta en la línea de la innovación educativa y calidad de vida para el desarrollo humano, ya que promueve la innovación como elementos de cambio individual, social y cultural, considerando la calidad de vida como principal indicador del desarrollo humano.

\section{Revisión Literaria: Antecedentes}

La capacidad de aprender y reaprender se constituye en la herramienta más valiosa de cualquier persona, ya que desde esa actitud que puedan irse adquiriendo las competencias necesarias según el espacio laboral en donde se encuentre, acudiendo a las teorías cuando se requiera, adoptando métodos idóneos y, sobre todo, innovando en la manera de resolver y enfrentar situaciones específicas (Ruiz et al., 2004).

En la actualidad se considera que las variables cognitivas, metacognitivas y conductuales son determinantes para la obtención del aprendizaje, sin embargo, es importante considerar una nueva variable que la modalidad utilizada en el proceso de aprendizaje (a distancia o presencial) considerando que cada una de ellas implica distintas influencias sobre el proceso de aprendizaje. Por ello se realizan algunos estudios Suárez y Amaya (2006) como el trabajado en Madrid con 298 estudiantes de las dos modalidades en donde se obtiene como resultados que los alumnos de modalidad a distancia están más interesados por aprender y dominar las tareas, se consideran a sí mismos más capaces de controlar el proceso de aprendizaje, así como más eficaces tanto en el desarrollo de su aprendizaje, así también se obtiene que el nivel de aprendizaje con otros compañeros es significativamente menor.

Se indaga lo relacionado a la educación por competencias su significado y sus orígenes, encontrándose con que se inicia en este enfoque de formación en México en la educación básica y media superior, al responder al plan nacional de desarrollo 2007-2012 considerándose los contextos sociales, políticos y económicos del país e internacionales.

El rápido desarrollo de la tecnología de la comunicación e información menciona (Martínez, 2008) ha generado cambios en el proceso de enseñanza aprendizaje al incorporar el uso de la tecnología en la enseñanza a distancia como en la presencial, y el buen uso de estas herramientas, mejora la productividad e impulsa el desarrollo, y donde este sistema tecnológico bidireccional y multidireccional no tiene fronteras en el tiempo por lo tanto, se pude dar en cualquier lugar.

Así mismo dice (García, 2001) la enseñanza presencial como está organizada no puede atender a todos, ni todos tienen la posibilidad de manejarse de esta manera por diferentes circunstancias, pero aprovechando el mayor avance de la tecnología se pueda recurrir a la educación a distancia 
como un medio alternativo y complementario, teniendo como principal característica a la flexibilidad que permite que más personas y sobre todo a los adultos adquieran conocimiento, considerando también que el alumno está consiente del mayor grado de responsabilidad que asumirá.

\section{Educación presencial}

El martes 21 de octubre del 2008 se publica en el diario oficial el Acuerdo 445 por el que se conceptualizan y definen para la educación media superior las opciones educativas en las diferentes modalidades de donde se desprende la conceptualización de la educación presencial,"Esta opción de la modalidad escolarizada se caracteriza por la existencia de coincidencias espaciales y temporales entre quienes participan en un programa académico y la institución que lo ofrece (SEP, 2008).

1. Aprenden en grupo. Por lo menos $80 \%$ de sus actividades de aprendizaje las desarrolan bajo la supervisión del docente.

2. Siguen una trayectoria curricular preestablecida.

3. Cuentan dentro del plantel con mediación docente obligatoria.

4. Pueden prescindir de la mediación digital.

5. Tienen en el plantel un espacio de estudio fijo.

6. Deben ajustarse a un calendario y horarios fijos.

7. Están sujetos a las evaluaciones que para acreditas los programas de estudio aplique la institución educativa.

8. Deben cumplir y acreditar el plan y programas de estudio para ser objeto de certificación.

9. Obtienen de la institución educativa el documento de certificación correspondiente.

Castellanos y Morga (2013) menciona con las palabras de Marissa Ramírez que desde el constructivismo la educación basada en competencias "extiende la necesidad de lograr en los estudiantes la transferencia de los conocimientos, no solo a contextos inmediatos si no a la vida misma, a lo que viven los estudiantes aquí y ahora, pero también a lo que tal vez necesiten para poder potenciar su vida futura. Se habla entonces de contenidos que tienen significado integral para la vida".

Gimeno, Perea, y Martinez (2008) menciona en su libro: Educar por competencias ¿qué hay de nuevo? Que:

1. La organización del aprendizaje por competencias pretende consolidar lo que se aprende dándole algún tipo de funcionalidad

2. Una orientación más precisa de este enfoque utilitarista de la enseñanza lo representan, las experiencias de formación profesional, en las que el dominio de determinadas destrezas, habilidades o competencias es la condición primordial del sentido de la formación. 
Un gran número de instituciones se rigen en la educación por competencias, es si como El Sistema Nacional de Institutos Tecnológicos menciona en su dimensión académica. - Que asume los referentes teóricos de la construcción del conocimiento, del aprendizaje significativo y colaborativo, de la mediación y la evaluación efectiva y de la práctica de las habilidades adquiridas, que se inscriben en dos perspectivas psicopedagógicas: sociocultural y estructuralista (Acosta, Armendariz, \& Bernal, 2012).

Jaques Delors la Organización de las naciones unidas para la educación y la cultura (UNESCO) a través del informe publicado en el año 1996 por Jaques Delors al que título la educación encierra un tesoro. La UNESCO puso de manifiesto la importancia de implementar una educación permanente a lo largo de la vida, que sea flexible, diversa y accesible. Alfa Tuning América Latina: Innovación Educativa y Social (2011-2013) busca continuar con el debate ya iniciado con la primera parte de este proyecto llevada a cabo de 2004-2008. Esta propuesta parte de un terreno ya abonado fruto del desarrollo de la fase anterior de Tuning América Latina (2004 - 2008) y ante una demanda actual de las universidades latinoamericanas y los gobiernos de facilitar la culminación del proceso iniciado. Tuning puso en el centro de la escena la importancia de las competencias para los procesos de modernización y reforma curricular.

A la vez la UNESCO también manifiesta la importancia de establecer una educación permanente y de por vida que se distinga por ser flexible, diversa y accesible y que se estructure en torno a cuatro aprendizajes fundamentales considerados como pilares del conocimiento Aprender a conocer, aprender a hacer, aprender a vivir juntos y aprender a ser descrito en el informe Delors en 1996, llamado aprender es un tesoro.

En trabajos anteriores, los autores han examinado las tendencias tecnológicas que han abierto nuevas oportunidades para las universidades electrónicas y siguió esto con el primer vistazo a los procesos clave involucrados en el paso de la impartición de conferenciantes físicos a virtuales. Este documento analiza los procesos de valoración y evaluación manifestados en exámenes con una mirada específica a los exámenes en línea como un trampolín clave hacia universidades electrónicas totalmente fiables. Para (Alarcón, Cervantes, \& Herrera, 2012) la educación a distancia está en constante proceso de expansión y de cambio es el ámbito en el cual se experimentan nuevos desarrollos de modelos educativos

La educación a distancia tiene una enorme progresión en lo que se refiere a la educación de trabajadores para mejorar de manera sensible sus competencias profesionales (Ruiz, 2009; Moreno, 2012). Concibo el desarrollo de la educación a distancia en todo su potencial, incorporada a las políticas y estrategias educativas integrales, como parte esencial de otras políticas sociales. Su fortaleza, necesariamente, va ligada a políticas de largo 
alcance que consideren la diversificación de oportunidades educativas; que con una visión más allá de lo escolar. Barud (2011) menciona la inversión en la educación a distancia empieza a adquirir un papel determinante para el futuro de los jóvenes en México, pues representa una opción para aquellos jóvenes que no cuentan con los recursos para cubrir con los gastos que implica asistir a la universidad

También es importante destacar que la educación a distancia tiene como ventajas la flexibilidad de horarios, por lo que los jóvenes pueden utilizar el resto de su tiempo para empezar a introducirse en el campo de trabajo del área que hayan elegido

Varias universidades han buscado gradualmente automatizar o mover operaciones en línea. La Innovación, La interacción, la colaboración son elementos clave junto con las iniciativas impulsadas por la tecnología. De hecho, Tapscott y Williams (2010) sugieren medios generados por el usuario, redes sociales, Crowdsource y la producción entre pares para el nuevo concepto universitario utilizando la colaboración masiva es la nueva forma de colectivismo online. En una publicación sobre universidades electrónicas, Ismail et al. (2017) examinó la tecnología y tendencias en universidades electrónicas, así como el manejo de conferencias/lecciones en el contexto del panorama general de los procesos universitarios. En este sentido, el manejo de la evaluación es un elemento importante en el movimiento adelantar el concepto de e-universidad. Este artículo presenta una revisión de la literatura sobre el proceso de evaluación de las e-universidades. El documento utiliza dos estudios de caso únicos de física y procesos de manejo de evaluaciones virtuales. Luego, el documento reflexiona sobre las mejoras y limitaciones del paso hacia el aula virtual dentro de un proceso e-universitario.

Con el avance de Internet, Bergstrand y Savage (2013) sugieren que las universidades y las universidades están cada vez más motivadas hacia el formato de universidades electrónicas. En este contexto, virtual. Se puede implementar educación en línea, y se pueden ofrecer cursos con mayores inscripciones y presupuestos más reducidos. Una encuesta realizada por Allen y Seaman (2014) a 2.500 universidades que mostraron que 5,6 millones de estudiantes estaban matriculados en al menos una clase en línea.

En la literatura sobre educación superior se encuentran varios términos y conceptos para referirse a ellos en línea, por ejemplo, Los cursos en línea se conocen como aprendizaje a distancia (en línea), e-learning, e-universidades, universidades virtuales y educación basada en Web 2.0 (Safieddine et al., 2017). Todas estas formas electrónicas de instituciones de educación superior buscan y apoyan la misma misión fundamental de la universidad tradicional (es decir, la base del campus) como la docencia, servicios de investigación y sociedad (Burnett \& Natalia, 2010). 
La educación virtual fusiona los beneficios de la tecnología con la sabiduría del aprendizaje tradicional y con un diseño eficiente, desarrollo y entrega. En este contexto, Lee et al. (2003) sugieren que una universidad en línea necesita entregar todos los aspectos de la universidad convencional a la virtual o en línea entorno que incluye enseñanza, procesos, estructuras, lugar de trabajo, personal, administración, soporte, ayudas, valoraciones, evaluaciones y servicios. Por lo tanto, las universidades en línea deberían considerar incorporar una educación con adecuada metodología que se basa en gran medida en información avanzada, tecnología e inteligencia artificial (IA). El objetivo de estas universidades es ofrecer cursos online de calidad con la creación y distribución de contenido virtual, administración, asistencias, evaluaciones, exámenes, y aspectos de supervisión, calificación y evaluación (Wong, 2012). De hecho, todos los aspectos mencionados deben confiar en una comunicación interactiva entre los miembros y las partes de diferentes universidades, como instructores, alumnos/estudiantes, jefe de departamento, registros y unidad de examen. Según Tapscott y Williams (2010), es fundamental que exista un proceso de flujo de información, donde diferentes partes y tipos y formas de información podrían participar en el proceso de formación

Hay un mayor costo en la educación superior y una reducción relativa en el costo de tecnología en comparación. Bergstrand y Savage (2013) sugieren que ha habido un interés renovado en la universidad en línea para proporcionar diferentes formas de entrega de conocimientos, ayudar a las instituciones de educación superior a hacer frente a un aumento de la matrícula y a presupuestos más reducidos. La universidad electrónica puede seguir el enfoque de aprendizaje asincrónico o sincrónico (Feldman \& Zucker, 2015). Anteriormente, los investigadores de la e-universidad habían basado su trabajo en el asincrónico enfoque de aprendizaje, en el que se aceptaron las limitaciones demoradas de Internet. Wu y Bieber (2008) explican que el enfoque de aprendizaje asincrónico involucra herramientas como el correo electrónico, discusión, grupos de noticias, tablones de anuncios, y archivos adjuntos. En contraste, investigaciones recientes Los esfuerzos hacia la euniversidad se basan en el enfoque de aprendizaje sincrónico, como el trabajo de investigación realizada por Atoum et al. (2017), Al-Musharraf y Alkhattabi (2016), y Nerkar (2017). Este enfoque tiene como objetivo permitir que los grupos que están separados en el tiempo y el espacio se involucren en la producción activa de conocimiento compartido. El enfoque de aprendizaje sincrónico no es un término nuevo en la enseñanza. El aprendizaje sincrónico fue definido por (Gunawardena et al., 1997) como instrucción y colaboración en tiempo real a través de Internet.

El modo de enfoque de aprendizaje sincrónico en e-universidad, normalmente implica muchas herramientas. Las cuales incluyen chat en vivo, 
conferencias de audio y video, datos y compartir aplicaciones, compartir una pizarra, "levantar la mano" virtual y ver juntos presentaciones multimedia y presentaciones de diapositivas en línea. (Humanante Ramos \& Col, 2015) explican esta nueva modalidad de e-universidad donde la información y las tecnologías de comunicación avanzadas están integradas en el campo educativo en una nueva revolución. Una revolución en la forma en que concebimos las experiencias de aprendizaje en comparación con forma tradicional. Ofrece varias ventajas sobre el tradicional, como que ahorra tiempo de aprendizaje y reduce el costo de su educación. Puede brindar más flexibilidad, conveniencia e igualdad de oportunidades para los alumnos. También proporciona una enseñanza innovadora y ricos contenidos. métodos y formas de entrega a sus estudiantes, que pueden ir desde películas, animaciones, material accesible desde la web hasta materiales de realidad virtual (VR). Además, Humanante Ramos, y Col. (2015) sugieren que la tecnología puede ayudar a reducir el costo ambiental al ir recortando el uso de papel, así como el transporte. Por tanto, las herramientas de Internet, RV e IA pueden una infraestructura dominante para el intercambio de conocimientos entre las personas y una nueva generación de universidades virtuales (Safieddine, 2017). Jardines (2010) presenta una descripción y reflexión sobre algunas comparaciones que realizan algunos analistas sobre las principales diferencias encontradas entre la educación presencial y la educación a distancia en Nuevo León.

Morata, Sotelo, y Vales (2010) realizan una investigación en Sonora México con la participación de 85 estudiantes de las modalidades de estudio a distancia y escolarizado sobre habilidades computacionales, uso de plataforma tecnológica y actitudes hacia diseño del curso a distancia. La investigación correspondió a un diseño no experimental, transeccional, correlacional. (Navarro) Se realiza una investigación con alumnos de Maestría en Educación generación 1999-2002 con edades de 22 a 44 años de la Sede Tejupilco originarios de diferentes municipios del estado de México. Con algunos objetivos, entre ellos el de: Analizar los factores asociados a la eficiencia terminal exitosa en la modalidad de educación a distancia en la Universidad Virtual Del Tecnológico de Monterrey Campus Toluca (Alarcón, Cervantes, \& Herrera, 2012). La educación a distancia está en constante proceso de expansión y de cambio es el ámbito en el cual se experimentan nuevos desarrollos de modelos educativos.

La educación a distancia está en constante proceso de expansión y de cambio es el ámbito en el cual se experimentan nuevos desarrollos de modelos educativos (Alarcón, Cervantes, \& Herrera, 2012). 


\section{Objetivos}

En trabajos anteriores, los autores han examinado las tendencias tecnológicas que han abierto nuevas oportunidades para las universidades electrónicas y siguió esto con el primer vistazo a los procesos clave involucrados en el paso de la impartición de conferenciantes físicos y virtuales. Este documento analiza los procesos de valoración manifestados en encuestas, con una mirada específica a las clases en línea y de manera presencial que permitan conocer rulas diferentes precepciones y formas de trabajo de las modalidades, También para comparar las diferencias de los sujetos que estudian de estas dos formas -Presencial y Asincrónica- ayudando a conocer los elementos que se pueden aplicar de una manera que permita homogenizar el desempeño escolar entre las dos modalidades de enseñanza. Por ello se plantean los siguientes objetivos:

- Obtener las diferencias específicas entre Educación sincrónica y Asincrónica.

- Determinar cuáles son las diferencias específicas entre los dos tipos de enseñanza.

- Establecer planes de trabajo que sean específicos en los dos tipos de enseñanza.

- Dar a conocer esas diferencias a los maestros del Instituto Tecnológico para que realicen un cambio de estrategia necesaria en la forma de enseñanza.

\section{Material y Metodología las variables sus tipos}

4.1. La definición operacional de las variables se da como sigue:

Estas variables están medidas en escala de 0 a 10 y la forma de pregunta es la que se observa en la definición de las mismas.

En qué grado eres:

1. Tenaz Definido como capaz de conseguir tus objetivos.

2. Organizado Capacidad de organizar conocimientos.

3. Uso estrategias de aprendizaje: definido Capacidad de usar distintas estrategias de aprendizaje.

4. Activo. Definido con capacidad de trabajar para encontrar información.

5. Buscas recursos para complementar los temas Uso de recursos en libros y tics.

6. Superas los obstáculos Obtención de lo que necesita.

7. Tolerante a la frustración. sujeto que no se da por vencido.

8. Investigador. busca información en bases de datos científicas.

9. Preguntón realiza consultas con compañeros o maestros. 
10. Te expresas en forma oral. Sus expresiones orales son congruentes.

11. Te expresas en forma oral escrita. Se comprende lo que escribe.

12. Comprendes expresiones orales. Entiende lo que le explican.

13. Comprendes expresiones escritas. Entiende lo que encuentra escrito.

14. Manejas información de distintas fuentes. Capacidad de usar bases de datos o bibliográficos.

15. Extrae inferencias de los textos. Extrae información adecuado de bibliografía.

16. Construyes visiones integradoras de la realidad. Capacidad de Integrar a la realidad.

17. Actúas creativamente. Utiliza su creatividad personal.

18. Dialogas. Expresa su conocimiento con otras personas.

19. Tienes una actitud abierta. Entiende los puntos de vista diferentes.

20. Utilizas hábitos adecuados de estudio. Tiene hábitos formados de estudio.

21. Trabajas colaborativamente con otros, trabaja con compañeros.

22. Comprometido. Se involucra con sus estudios y trabajos.

23. Responsable.es cumplidor y sensato con sus trabajos.

24. Activo en el proceso educativo. Se compromete con sus procesos de educación.

25. Activo en la evaluación. Se prepara para su evaluación.

26. Se te facilita el aprendizaje colaborativo. Tiene facilidad para colaborar con aprendizaje.

27. Planificas la tarea. Utiliza tiempo para realizar sus trabajos.

28. Recapitulas los temas vistos. Realiza resúmenes.

29. Relacionas los temas vistos. Resume varios temas en uno solo.

30. Reorientas las experiencias de aprendizaje ve desde distintos puntos el aprendizaje.

4.2. Arreglo situacional de las variables (Kerlinger \& Lee, 2002).

Se establece el arreglo situacional y observacional de las variables que utiliza el investigador, su expresión gráfica y explicada informan al lector sobre cómo se ordenaron las variables para desarrollar la investigación. Este apartado también ubica sobre la operacionalidad, funcionalidad, ejecución y derivación expositiva del proceso de investigación, Además permite que se informe sobre la manera en que se va a analizar el fenómeno propio de la investigación, y como se darán respuestas a las preguntas de la misma. 


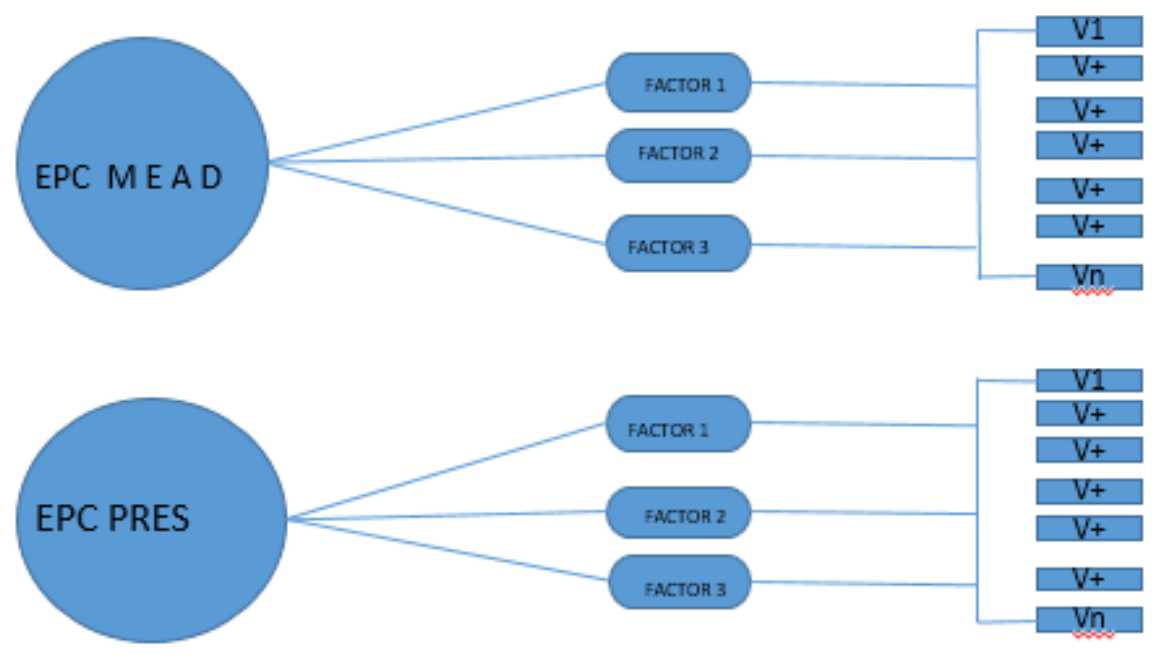

Fuente Elaboración propia

Explicación: EPC MEAD= significa Educación a Distancia, los factores están formados por las variables principales del Factor.

EPC PRES= significa Educación presencial, los factores están formados por las variables principales del Factor.

\subsection{Instrumento}

Se aplicó una encuesta a 106 sujetos de las dos modalidades de Estudio; a Distancia y Presencial con un porcentaje de 40.6. De sujetos de aprendizaje a Distancia y $50.4 \%$ de sujetos con estudios presenciales habiendo obtenido para cada muestra una confiabilidad en la encuesta de: grupo presencial con un alfa de Cronbach's; y Grupo a Distancia (Asincrónico) con .903 y grupo presencial de .919 .

Estadísticas de fiabilidad (Cochran William, 1984)

\begin{tabular}{|l|l|l|}
\hline $\begin{array}{l}\text { modalidad Modalidad de } \\
\text { estudo }\end{array}$ & $\begin{array}{l}\text { Alfa de } \\
\text { Cronbach }\end{array}$ & N de elementos \\
\hline 1 Presencial &, 919 & 30 \\
\hline 2 A Distancia &, 903 & 30 \\
\hline
\end{tabular}

Fuente: Elaboración propia.

\subsection{Procedimiento}

Tipología de la Investigación

La presente investigación es exploratoria, a partir de ella se obtendrá más información acerca de las habilidades que componen los campos de las competencias en dos modalidades de estudio de la cual una de ellas es relativamente joven y por tanto poco estudiada como es la educación a distancia, así como denotar las diferencias que presentan.

Descriptiva porque las variables simples realizan una agrupación que permite describir las características de cada modalidad de estudio dada de la 
agrupación y relación que se da entre ellas, así como a través de ello denotar las diferencias que se presentan.

Correlacional debido a que se buscan las relaciones significativas entre las variables tanto de estudio a distancia como estudio escolarizado.

\section{Resultados}

Se realiza un análisis de Factores, separados por tipos de Estudio Presencial y Asincrónica con Extracción de método de Componentes Principales, Rotación Varimax.

Tabla 1. Matriz de componente ${ }^{\mathrm{a}, \mathrm{b}}$

\begin{tabular}{|l|l|}
\hline \multirow{2}{*}{} & Componente \\
\cline { 2 - 2 } 2 tenaz &, 608 \\
\hline 3 organizado &, 639 \\
\hline 4 estrateg &, 619 \\
\hline 5 activo &, 509 \\
\hline 7 superas &, 690 \\
\hline 13 comprendeoral &, 720 \\
\hline 14 comprenescr &, 559 \\
\hline 15 manejainf &, 598 \\
\hline 16 extraeinfo &, 722 \\
\hline 17 constvision &, 684 \\
\hline 18 actuacreat &, 576 \\
\hline 20 actabierta &, 608 \\
\hline 21 habitdeest &, 720 \\
\hline 23 comprometi &, 806 \\
\hline 24 Responsab &, 829 \\
\hline 25 actproed &, 717 \\
\hline 26 activoeval &, 656 \\
\hline 28 planftare &, 527 \\
\hline 29 recatmsvts &, 702 \\
\hline 30relactemvis &, 597 \\
\hline 31reorientaex &, 663 \\
\hline
\end{tabular}

Método de extracción: análisis de componentes principales.

a. modalidad 1 Modalidad de estudio $=1$

Presencial

b. 1 componentes extraídos.

Fuente: Elaboración propia. 
Tabla 2. Matriz de componente ${ }^{\mathrm{a}, \mathrm{b}}$

\begin{tabular}{|c|c|}
\hline & Componente \\
\hline & 1 \\
\hline 4 estrateg & ,714 \\
\hline 6 buscarec &, 589 \\
\hline 7 superas & ,700 \\
\hline 9 investigador & ,764 \\
\hline 11 expresaoral &, 533 \\
\hline 13 comprendeoral &, 665 \\
\hline 14 comprenescr &, 693 \\
\hline 16 extraeinfo &, 614 \\
\hline 17 constvision &, 687 \\
\hline 19 dialogas &, 610 \\
\hline 20 actabierta & 655 \\
\hline 22 trabcolab &, 569 \\
\hline 23 comprometi &, 582 \\
\hline 25 Actproed &, 615 \\
\hline 26 activoeval &, 678 \\
\hline 27 facapcol & 699 \\
\hline 29 recatmsvts &, 583 \\
\hline 30 relactemvis & 655 \\
\hline 31 reorientaex & ,758 \\
\hline
\end{tabular}

Método de extracción: análisis de componentes principales.

a. modalidad 1 Modalidad de estudo $=2$

A Distancia

b. 1 componentes extraídos.

Fuente: Elaboración propia.

En la Tabla 1 y Tabla 2 los resultados son los siguientes; las variables que no se saturaron en los factores son: v8.- tolerante a la frustración; v10 Realizan consultas con maestros o compañeros; v12 se comprende lo que escribe; lo que señala que tanto los estudiantes que estudian en forma presencial como a distancia, No presentan problemas con frustración, relación educativa con maestros o compañeros y tienen capacidad de escribir para otras personas.

En relación con los dos métodos de enseñanza -presencial y no presencial- los alumnos presentan las características correspondientes a V4 capacidad de utilizar diferentes estrategias de aprendizaje; v7, superan obstáculos para obtener lo necesario para su estudio; V13 también tienen la capacidad de entendimiento de las explicaciones que se les proporcionan,v14 comprenden cuando utilizan la lectura para su estudio; v16 extrae información adecuada de los textos; v17 los alumnos construyen visiones integradoras de la realidad; v20 los alumnos presentan actitud abierta; v23 los alumnos son comprometidos ya que se involucran con sus estudios y trabajos; v25 son 
activos en el proceso educativo; v26, también son activos en su proceso de evaluación; v29 recapitulan temas vistos; v30 relacionan los temas vistos; v31 y reorientan las experiencias de aprendizaje. Todo lo anterior denota que los alumnos por el hecho de ser estudiantes, no importando la forma de aprendizaje, conforman su estrategia de aprendizaje de tal manera que les permite superar obstáculos en la comprensión oral y escrita, esto les ayuda a extraer información con una actitud abierta que les permite construir su propia visión de la realidad; lo que los obliga a recapitular y relacionar temas vistas, reorientando su trabajo e involucrarse en el proceso de aprendizaje, volviéndolos activos en el proceso de aprendizaje y en los procesos de evaluación.

En la enseñanza presencial los estudiantes presentan tenacidad, organización activos o trabajadores, con actuación creativa y responsabilidad, con hábitos de estudio, lo que les permite establecer tiempos de estudios con la que planifican sus tareas y les da el estatus de responsables, lo que muestra que la educación presencial permite que el alumno sea más eficiente y laborioso derivado de los procesos de enseñanza que el maestro proporciona de manera guiada.

En la enseñanza asincrónica o a distancia, los alumnos presentan rasgos de búsqueda de recursos, tienen que aprender a expresarse oralmente para poder realizar diálogos con maestros y compañeros, por lo que tienen que desarrollar en gran medida el trabajo colaborativo cuando se presentan en asesoramiento o dirección de las personas que trabajan con ellos, debido a que deben replantear y hacer comprender a sus interlocutores en lo que desean tener claridad en las actividades de investigación de los temas de sus tareas. Esto revela que el trabajo que los alumnos a distancia deberán realizar presenta un grado un poco más alto de dificultad para comprender eficientemente sus conocimientos.

\section{Conclusiones}

Los estudiantes que estudian en forma presencial como a distancia, No presentan problemas con frustración, relación educativa con maestros o compañeros y tienen capacidad de escribir para otras personas. Esto refleja que los dos tipos de enseñanza presentan en el alumno un grado de normalidad en sus relaciones personales y que tienen habilidades de comunicación. Esto probablemente es debido a que en la era de la información, las nuevas formas de Tecnologías de Información y Comunicación ya son comunes para los alumnos y para los maestros, sin embargo, estos canales de comunicación deben satisfacer las necesidades y expectativas de los estudiantes y de los maestros; ya que hay una diferencia importante en la utilización, debido a que, maestros y alumnos tienen fines diferentes para el manejo de las aplicaciones; 
unos son para comunicación y diversión y otras cosas, y los otros para validación y aplicación de conocimientos con softwares especializados.

Aquellos estudiantes, no importando la forma de aprendizaje, presencial o a distancia- tienen en común que conforman su estrategia de aprendizaje de tal manera que les permite superar obstáculos en la comprensión oral y escrita, esto les ayuda a extraer información con una actitud abierta que les permite construir su propia visión de la realidad; lo que los obliga a recapitular y relacionar temas vistos, reorientando su trabajo e involucrarse en el proceso de aprendizaje, volviéndolos más activos en el aprendizaje y en los procesos de evaluación, lo que muestra que la educación presencial permite que el alumno sea más eficiente y con más laboriosidad, derivado de los procesos de enseñanza que el maestro proporciona de manera guiada, y de manera asincrónica.

Los alumnos que estudian de manera presencial presentan capacidad de tenacidad, organización activos o trabajadores, con actuación creativa y responsabilidad, y hábitos de estudio, lo que les permite establecer tiempos de estudios con la que planifican sus tareas y les da el estatus de responsables, lo que muestra que la educación presencial permite que el alumno sea más eficiente y laborioso derivado de los procesos de enseñanza que el maestro proporciona de manera guiada

Lo anterior sustenta lo que Nuñez y Vigo (2014) mencionan en el 2014 que la educación por competencias se trabaja desde los años de 1980 en delante es en los años 90 cuando empieza a tomar auge ya que la misma UNESCO menciona su importancia través del informe que realiza DELORS en su informe que lleva por nombre la educación es un tesoro en donde se menciona la importancia de una educación por competencias a la que considera una educación para la vida y que está fundamentada en cuatro pilares que son : aprender a conocer, aprender a hacer, aprender a vivir juntos y aprender a ser.

En la enseñanza asincrónica o a distancia, los alumnos presentan rasgos de búsqueda de recursos, les exige a expresarse oralmente para poder realizar comunicación con integrantes de esta forma de enseñanza, por lo que tienen que desarrollar en gran medida el trabajo colaborativo tanto con compañeros como con asesores, para ellos es necesaria la dirección de las personas que trabajan con ellos, debido a que deben replantear y hacer comprender a sus interlocutores con claridad en las actividades de investigación de los temas de sus tareas. Esto revela que el trabajo que los alumnos a distancia deberán realizar presenta un grado un poco más alto de dificultad para comprender eficientemente sus conocimientos. El aprendizaje Asincrónico, revela que probablemente el trabajo que los alumnos a distancia deberán realizar presenta un grado un poco más alto de dificultad para comprender eficientemente sus conocimientos. 
En las habilidades de los jóvenes estudiantes asincrónicos encontradas en este trabajo se encuentra en alta relación con lo que expresa (Suarez \& Anaya, 2006) donde menciona que en su resultados estos jóvenes, son eficaces tanto en el desarrollo de su aprendizaje como en el resultado, ya que como (Maya, 1993) destaca, el estudio independiente presenta el alto grado de interés, responsabilidad, empeño y cierto control de los aprendizajes (Jardines, 2010) menciona que a mayor interacción con el profesor, y con el contenido y su relación con otros alumnos, enfatiza la autonomía del estudiante. Morata (2010) considera el aprendizaje significativo y la participación de manera más activa, autoeficacia, realización de tareas y exámenes, son de gran utilidad, de lo visto en clase, lo que permite auto control del aprendizaje, de esta manera los alumnos reconocen su esfuerzo y responsabilidad, como lo señala Navarro al definirlos como disciplinados, organizados, con administración de tiempos capacidad de aprendizaje por cuenta propia se generan habilidades de aprender a aprender.

Para finalizar las conclusiones están acordes con las teorías presentadas en el trabajo de investigación realizado. Lo que permite determinar que hay diferencias significativas entre las personas que estudian de manera asincrónica con las personas que lo hacen de manera presencial. Este trabajo solo presenta características descriptivas y relacionadas con el comportamiento estudiantil. Por lo que se recomienda hacer más estudios para generar la utilización de las capacidades de estudio y homogeneizar los dos tipos de enseñanza.

\section{References:}

1. Acosta, G. M., Armendariz, B. G., \& Bernal, N. A. (2012). Modelo educativo para el siglo XXI Formacion y desarrollo de Competencias Profesionales (1a. edición ed.). Mexico; DF, Distrito Federal, México: Esfera, Crativa. Recuperado el Noviembre de 2016.

2. Alarcón, R., Cervantes, F., \& Herrera, A. (2012). Las nuevas fronteras de educación a distancia. Obtenido de http://virtualeduca.org/documentos/observatorio/oevalc_2012_(fronte ras).pdf

3. Barud, R. S. (2011). comunidad.unam.mx. Obtenido de comunidad.unam.mx:

http://www.cuaed.unam.mx/encuentro_2011/encuentrocu/pdf/fe/sami aguadalupe.pdf

4. Castellanos, T. N. \& Morga, R. L. (2013). Educacion por competencias hacia la excelencia en la formación superior (1a. ed.). Tlalnepantla, Estado de Mexico, Mexico: Red Tercer Milenio. Recuperado el Noviembre de 2016, de https://www.cife.edu.mx/Libros/7/educacion_por_competencias.pdf 
5. Cochran William, G. (1984). Técnicas de Muestreo (4a. Impresión). México: Ed Cia. Editorial Continental, S.A. (C.E.C.S.A.).

6. Florido, B. R. \& Florido, B. M. (2003). Etic@ Net. Obtenido de Etic@Net:

http://www.ugr.es/ sevimeco/revistaeticanet/Numero1/Articulos/EaD retos.pdf

7. García, A. L. (2001). Educacion a distancia ayer y hoy. Obtenido de Quaderns digitals.net: www.quadernsdigitals.net/datos_web/1_1400/en Linea/10.pdf

8. Gimeno, S. J., Perea, G. A., \& Martinez, R. J. (2008). Educar por competencias ¿que hay de nuevo? (978-7112-528-6 ed.). Madrid, España: Morata. Obtenido de http://galeon.com/chanram/parte1.pdf

9. Hair, F. B. R. (2010). Investigación de Mercados En un Ambiente de Información Digital (4a. Edición). México : Ed. McGraw-Hill.

10. Hair, f. J., Anderson, R., Tatham, R., \& Black, W. C. (2008). Análisis Multivariante (5a. ed.). Madrid: Pearson prentice Hall. Recuperado el noviembre de 2016

11. Hernandez, S. R., Fernandez, C. C., \& Baptista, L. P. (2006). Metodologia de la investigación (4a. ed.). Iztapalapa, Mexico, México: Mc Graw Hill. Recuperado el Octubre de 2016

12. Jardines, F. J. (2010). La educacion a distancia una comparació con la educación presencial, en relación con la función del profesor, del estudiante $y$ los medios. Obtenido de http://www.web.facpya.uanl.mx/rev_in/Revistas/7.2/A9.pdf

13. Kerlinger, F. N. \& Lee, H. B. (2002). Investigación del Comportamiento, Técnicas y Metodología (4a Edición). México: McGraw-Hill.

14. Kerlinger, F. \& Howard, L. (2002). Investigacion del comportamiento. métodos de Investigación en Ciencias Sociales. México: Mc Graw Hill.

15. Levin, J. (1979). Fundamentos de estadistica en la investigación social. Mecico, D.F, Mexico: Harla. Recuperado el Noviembre de 2006

16. Martínez, U. C. (2008). Educacion a distancia: sus caracteristicas y necesidad en la educacion actual. Obtenido de http://revistas.pucp.edu.pe/index.php/educacion/article/view/1532/14 77

17. Maya, B. A. (1993). Orientación basica sobre educacion a distancia y la funcion tutorial. San Jose, Costa Rica: UNESCO SAN JOSE.

18. Morata, M. L., Sotelo, M. A., \& Vales, J. (2010). Desarrollo de competencias en entornos educativos a distancia. Obtenido de 
http://www.itson.mx/publicaciones/Documents/cienciassociales/desarrollodecomptencias.pdf

19. Moreno, C. M. (2012). Veinte Visiones de la educación a distancai. Guadalajara: EDG virtual. Obtenido de http://biblioteca.udgvirtual.udg.mx/eureka/pudgvirtual/20_visiones_ web.pdf

20. Navarro, R. E. (s.f.). RED, Revista de educación a distancia, 12. Obtenido de RED, Revista de educación a distancia, 12: http://www.um.es/ead/red/12/edel.pdf

21. Nunnally, J. C. (1987). Teoría Psicométrica. México: Ed. Trillas .

22. Nuñez, N. \& Vigo, O. (2014). Formación universitaria basada en competencias. Chiclayo: FORMATS PRINT.

23. Ruiz, C. M. (2009). Concepción y Tendencias de la educación a distancia en America Látina. Madrid: Centro de Altos Estudios Universitarios de la OEI. Obtenido de DOCUMENTO2caeu.pdf

24. Ruiz, D. I., Enriquez, A. A., \& Ortiz, B. A. (2004). La innovación en la educación superior. México: ANUIES. Recuperado el noviembre de 2016, de https://comitecurricularsistemas.wikispaces.com/file/view/INNOVA CION_EN_LA_EDUCACION_SUPERIOR_ANUIES_MAYO+200 4.pdf

25. SEP (2008). ACUERDO número 445 por el que se conceptualizan y defi. Diario Oficial, 1-5. (G. Federal, Ed.) Distrito Federal, Distrito Federal, México: Gobierno Federal. Recuperado el Enero de 2017, de https://www.sep.gob.mx/work/models/sep1/Resource/7aa2c3ff-aab8479f-ad93-db49d0a1108a/a445.pdf

26. Suarez, A. J. \& Anaya, N. D. (2006). Educacion a distancia y presencial, Diferencias en los componentes cognitivos $y$ motivacionales de estudiantes universitarios. Obtenido de http://www.biblioteca.org.ar/libros/142095.pdf 